\title{
BEST-EFFORT COOPERATIVE COMMUNICATION WITHOUT DEDICATED RELAYS
}

\author{
Nate Goergen $\quad$ K. J. Ray Liu T. Charles Clancy \\ Department of Electrical and Computer Engineering \\ University of Maryland \\ College Park, MD 20742
}

\begin{abstract}
Traditional decode-and-forward cooperation systems consider dedicated relays, while instead we consider wireless transceivers that cooperatively relay signals in addition to primary communication missions. A system that transmits the additional signal using a best-effort transmission policy within the original transmission energy constraint is considered. To maintain the original energy budget we consider the feasibility of reallocating energy from pilot signals toward the relaying service when channel conditions are stationary. Under the best-effort delivery policy, the node is not obligated to devote energy for relaying signals, nor does it provide a guarantee of signal quality to retransmissions. Instead the relay sacrifices energy at its own discretion, prioritizing the primary communication mission. Using the best-effort delivery policy, we derive an optimal power allocation rule that maintains a fixed symbol error rate for the relay's primary transmission, and further demonstrate cooperative communication gains using the proposed delivery method.
\end{abstract}

Index Terms - Cooperative systems, Radio repeaters, Communication channels, Communication system performance, MIMO systems

\section{INTRODUCTION}

Cooperative communication through the judicious use of relay nodes has proven extremely advantageous in decreasing outage probabilities when the channel between the source and destination is of poor quality [1]. Traditional relay schemes typically consider only nodes dedicated to users and nodes dedicated to relay services. However, when a dedicated relay is not present, nodes with their own communication mission must assist in relaying signals. We consider a relaying system where nodes provide relay services in addition to primary communications, that is, the relay service is provided on a best-effort basis.

Under the proposed scheme, a node with its own communication mission may also offer relay services while maintaining an energy budget. The best-effort nature of this system implies that the relay node is under no obligation to devote energy for relaying signals, nor does it provide a guarantee of signal quality for the relayed signal. A diagram depicting the best-effort relay system and the relationship between the relay-user and primary-user channels is depicted in Figure 1.

As a motivating example for adaptive best-effort relaying we consider the scenario of stationary channel conditions, when less energy is required for channel estimation purposes. The relay may choose to devote more power toward relaying services and less power toward channel estimation devices, according to the quality of service (QoS) requirements of the primary-user. When channel conditions require additional channel estimation energy, as is the case when the primary-channel is undergoing change, the relay may choose to allocate additional power toward the pilot component of the transmission for channel estimation purposes and less power for relay transmissions. The proposed best-effort delivery method introduces a unique power allocation problem, in which the relay must select optimal power allocation between pilot and relay-user signals, and in doing so provides one example of how transmission energy may be scavenged to achieve cooperative diversity.

While many pilot-embedding techniques have been discussed, we formulate our best-effort delivery channel using the pilotembedding framework proposed in [2], which generalizes how pilot signals may be embedded into data signals using a Space Time Code (STC) approach. With this embedding method, mutually orthogonal pilot and data signals are combined as a composite STC block before transmission. The STC approach is used as a general method for defining a sequence of symbols with periodically occurring pilot signals comprising a single block code, and may describe systems with one or more transmit antenna. The method easily extends to broadband signals such as ODFM, through the Space Time Frequency (STF) block code model [3]. Here, we investigate best-effort cooperative relaying using generic pilot embedding when the data-component of a data-bearing pilot block is reserved for relay transmissions.

In this paper we derive optimal power allocation policies with respect to the Symbol Error Rate (SER) of the primary-user, which will be used as a measure of QoS for the primary signal.

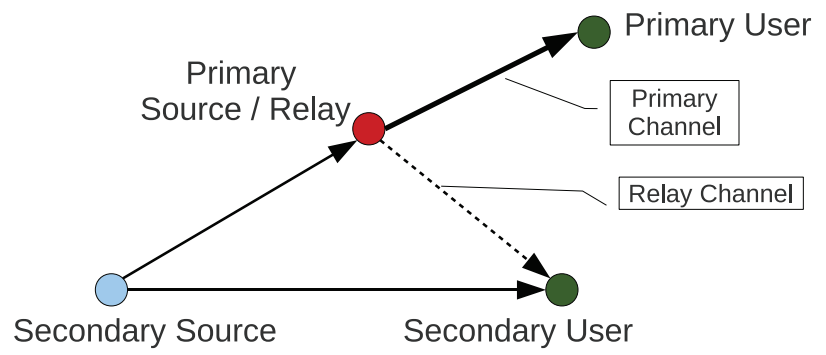

Fig. 1. System diagram of a best-effort relay system

This paper is orginized as follows: Section 2 generalizes databearing pilot frameworks and briefly introduces previous powerallocation work with relay channels. In section 3 the best-effort relay problem is discussed, and optimal power allocation with respect to the primary-user is derived. In section 4 we conclude with brief observations of the proposed best-effort relaying system. 


\section{SYSTEM MODEL AND PROBLEM FORMULATION}

We briefly describe the channel model used in our best-effort delivery scheme. Consider the communication system with $L_{t} \geq 1$ transmit antenna(s) and $L_{r} \geq 1$ receive antenna(s), with a Space Time (ST) block transmitted at index $t$ described by matrix $\mathbf{U}(t)$ of size $L_{t} \times M$. The $\mathrm{ST}$ block $\mathbf{U}(t)$ is transmitted across all $L_{t}$ transmit antennas in $M$ time slots. The received block $\mathbf{Y}(t)$ expressed in matrix form is:

$$
\mathbf{Y}(t)=\mathbf{H}(t) \mathbf{U}(t)+\mathbf{N}(t)
$$

with the channel coefficient matrix $\mathbf{H}(t)$ of size $L_{r} \times L_{t}$ describing the channel conditions experienced by the block at time $t$. The channel noise $\mathbf{N}(t)$ is modeled as complex white Gaussian noise with zero mean and variance $\left(\sigma^{2} / 2\right) \mathbf{I}(t)_{\left(L_{r} M \times L_{r} M\right)}$. We assume the elements of $\mathbf{H}(t)$ to be independent Rayleigh fading and quasi-static, where $\mathbf{H}(t)$ remains constant over each symbol block. The channel estimation problem is to estimate $\mathbf{H}(t)$ and recover the original ST block $\mathbf{U}(t)$, where the channel estimate is computed from the pilot / training signals embedded in the original transmission.

Using the pilot-embedding framework presented in [2], a composite transmission is composed of a data-bearing pilot partition and an ordinary ST coded signal partition. We assume the density of the pilot signals and bandwith resoruces for each partition to be constant. Thus, the bandwidth resources allocated for primary-user and relay transmissions are also constant. The data matrix $\mathbf{Z}(t) \in \mathcal{C}^{L_{t} \times M}$ is constructed as

$$
\mathbf{Z}(t)=\mathbf{D}(t) \mathbf{A}
$$

with ST code data-bearer matrix $\mathbf{D}(t) \in \mathcal{C}^{L_{t} \times N}$ and data-projection matrix $\mathbf{A} \in \mathcal{R}^{N \times M}$. Here, $N$ is the number of time slots reserved for data transmission, while time slots $M-N, N<M$ are reserved for the embedded pilot signals. The ST matrix is assumed to follow the constraint $E[\|\mathbf{H}(t)\|]^{2}=L_{t}$, or constant energy under the Frobenius norm. The augmented ST symbol to be transmitted becomes

$$
\mathbf{U}(t)=\mathbf{Z}(t)+\mathbf{P}=\mathbf{D}(t) \mathbf{A}+\mathbf{P}
$$

where $\mathbf{P} \in \mathcal{R}^{L_{t} \times M}$ is the pilot matrix. The salient point of the databearing framework is that pilot-embedding schemes may be generalized through the superposition of the data-bearing structure $\mathbf{D}(t) \mathbf{A}$ and the pilot matrix $\mathbf{P}(t)$. The data-projection and pilot matrix satisfy the following properties

$$
\begin{aligned}
\mathbf{A} \mathbf{P}^{T} & =\mathbf{0} \in \mathcal{R}^{N \times L_{t}} \\
\mathbf{P A}^{T} & =\mathbf{0} \in \mathcal{R}^{L_{t} \times N} \\
\mathbf{A} \mathbf{A}^{T} & =\beta \mathbf{I} \in \mathcal{R}^{N \times N} \\
\mathbf{P P}^{T} & =\alpha \mathbf{I} \in \mathcal{R}^{N \times L_{t}}
\end{aligned}
$$

where $\beta$ is a real-valued power of the data signal, and $\alpha$ is a realvalued power of the pilot portion of the signal. The $\alpha$ term will become an important parameter, as it represents the fraction of divertable power that is retained for pilot signals. The properties (4) through (7) of the data-projection matrix $\mathbf{A}$ and $\mathbf{P}$ essentially allow A to project the data component $\mathbf{D}(t)$ onto the orthogonal subspace of the pilot matrix $\mathbf{P}$, allowing for signal demodulation by means of a Maximum Liklihood (ML) receiver. Under the assumption that the pilot structures of (4) through (7) operate under a power constraint,

$$
\beta=P_{p}-\alpha
$$

with $P_{p}$ being the original normalized block transmission power of the node. According to (8) the power allocated to the composite signal consisting of the pilot plus relay-user data signal is equal to the power of the original node transmission. The signal at the receiver becomes

$$
\mathbf{Z}(t)=\mathbf{D}(t)(\mathbf{D}(t) \mathbf{A}+\mathbf{P})+\mathbf{N}(t)
$$

Three basic structures are discussed in [2] for the design of $\mathbf{A}$ and $\mathbf{P}$, including the Time-Multiplexed (TM) structure which generalizes the previous PSAM pilot embedding techniques. The PEP according to Chernoff's upper bound with respect to independent Rayleigh distributed channel and the PEP expression when channel information is known by the receiver are presented in [4].

In Decode-and-Forward (DF) relaying, a relay first receives and then decodes the signal from the source node before retransmitting the signal to the destination. We compare the optimal allocations obtained for best-effort relay delivery presented to the optimal allocations obtained for fixed DF relaying using dedicated relays, presented in [5]. We formulate the power optimization problem as finding the minimum pilot power factor $\alpha$ that maintains a minimum level of QoS with respect to the primary user, denoted $G_{p}$, according to some QoS rule $g(\alpha, \eta)$, that is

$$
\min _{\alpha} g(\alpha, \eta) \geq G_{p}, \quad 0 \leq \alpha, \eta \leq 1
$$

where $\eta$ is the primary-user's channel estimate confidence factor, to be defined in the next section.

\section{ANALYSIS OF BEST-EFFORT DELIVERY}

In this section we derive the pairwise error probability, and optimal power allocations for the best-effort relay channel. We assume that the number of timeslots $M$ in the $\mathrm{ST}$ transmission $\mathbf{U}(t)$ remains constant, and the number of timeslots used for data transmission $N$ also remains constant. Using this criteria the number of timeslots available for best-effort transmissions also remains constant.

Let us consider the case where channel between the relay and the primary-user, depicted as the solid line in Figure 1, is stationary over at least two consecutive blocks and the primary-user is able to detect this event. Such conditions may occur between fixed or immutable nodes, and in this motivating example we assume that transmission of additional pilot signal energy will not dramatically alter or improve the receiver's channel estimate. We assume that the primary-user channel estimate $\hat{\mathbf{H}}_{p}(t)$ has perfectly estimated the channel $\mathbf{H}_{p}(t)$, that is

$$
\hat{\mathbf{H}}_{p}(t)=\mathbf{H}_{p}(t)
$$

Under (11), the PEP for the primary-user becomes

$$
P(\mathbf{d} \rightarrow \mathbf{e})_{\mathbf{H}_{p}(t)} \leq\left(\prod_{i=1}^{L_{\Delta_{p}}} \lambda_{i_{p}}\right)^{-L_{r_{p}}}\left(\frac{P_{p}}{4 \sigma_{p}^{2}}\right)^{-L_{\Delta_{p}} L_{r_{p}}}
$$

where $L_{\Delta_{p}}$ is the rank of the primary-user's channel $\mathbf{H}_{p}(t), \lambda_{i_{p}}$ are the eigenvalues of the primary-channel, $L_{r_{p}}$ are the number of receive antennas used at the primary-user's destination, and $P_{p}$ is the normalized power used by the primary-user for transmission. The PEP for the relay-user may be expressed as

$$
\begin{aligned}
& P(\mathbf{d} \rightarrow \mathbf{e})_{\hat{\mathbf{H}}_{s}(t)} \leq \\
& \left(\prod_{i=1}^{L_{\Delta_{s}}} \lambda_{i_{s}}\right)^{-L_{r_{s}}}\left(\frac{1+\frac{\sigma_{s}^{2}}{\alpha}}{\frac{4 \sigma_{s}^{2}}{N}\left(\frac{N}{P_{p}-\alpha}+\frac{L_{t_{s}}}{\alpha}\right)}\right)^{-L_{\Delta_{s}} L_{r_{s}}}
\end{aligned}
$$


where $L_{\Delta_{s}}$ is the rank of the the channel $\mathbf{H}_{s}(t)$ between the the relay and the relay-user, $\lambda_{i_{s}}$ are the eigenvalues of the relay-channel, $L_{r_{s}}$ are the number of receive antennas used at the relay-user's destination, and $P_{p}$ is the normalized power used by the primary-user for transmission. We note that in this situation, the node is preforming primary mission transmissions while concurrently acting as a relay for the relay-user.

Under the proposed best-effort delivery policy, power for channel estimation purposes is diverted by the primary-user toward relayuser transmissions. It should be noted that the relay-user also requires energy for proper channel estimation, thus there exists a performance tradeoff for the relay-user as power to the pilot signals is decreased. To understand the behavior of the power allocation term $\alpha$ in terms of relay-user performance, we first note that the normalized block power may be expressed as (14) [2].

$$
\begin{aligned}
P_{s} & =\frac{E\left[\|\mathbf{U}(t)\|^{2}\right]}{L_{t}}=\frac{E\left[\|\mathbf{D}(t) \mathbf{A}\|^{2}\right]}{L_{t}}+\frac{E\left[\|\mathbf{P}\|^{2}\right]}{L_{t}} \\
& =\beta+\alpha=1
\end{aligned}
$$

We now look at the optimal power allocation for the pilot-part and data-part of the proposed scheme, and how this criteria may change with respect to the needs of the primary-user. Since the primary-user may use its prior channel estimate when current channel conditions do not warrant re-estimation of the channel, the PEP for the primary-user does not depend directly on $\alpha$ because pilot signals are ignored in this case. However, for each block transmission there is a chance that channel conditions may change requiring the primary-user to update its channel estimate using the pilot signal embedded in the transmission. We model this scenario in simple probabilistic terms as a two-state model given as

$$
\nu= \begin{cases}0, & \hat{\mathbf{H}}_{p}(t-1)=\hat{\mathbf{H}}_{p}(t)=\mathbf{H}_{p}(t) \\ 1, & \hat{\mathbf{H}}_{p}(t-1) \neq \mathbf{H}_{p}(t)\end{cases}
$$

In state $\nu=0$, the primary channel is modeled as stationary. Thus the channel estimate for the current code remains unchanged from the prior code estimate, which has perfectly estimated the channel. The PEP for the primary-user may be expressed as

$$
P(\mathbf{d} \rightarrow \mathbf{e} \mid \nu=0)_{\mathbf{H}_{p}(t)} \leq\left(\prod_{i=1}^{L_{\Delta_{p}}} \lambda_{i_{p}}\right)^{-L_{r_{p}}}\left(\frac{P_{p}}{4 \sigma_{p}^{2}}\right)^{-L_{\Delta_{p}} L_{r_{p}}}
$$

We define a new model parameter $\eta$

$$
\eta=P(\nu=0)=1-P(\nu=1)
$$

The parameter $\eta$ is the probability that the receiver's channel estimate will not require updating from the previous estimate, and will be described as the primary-user's channel estimate confidence. We assume $\eta$ to be estimated by the primary-user and available to the relay via a control channel.

When the channel state is $\nu=1$, channel conditions for the primary-user have changed substantially, requiring the receiver to update its channel estimate $\hat{\mathbf{H}}_{p}(t)$. In this state the receiver will experience a PEP from (13) expressed as

$$
\begin{aligned}
& P(\mathbf{d} \rightarrow \mathbf{e} \mid \nu=1)_{\hat{\mathbf{H}}_{p}(t)} \leq \\
& \left(\prod_{i=1}^{L_{\Delta_{p}}} \lambda_{i_{p}}\right)^{-L_{r_{p}}}\left(\frac{1+\frac{\sigma_{p}^{2}}{\alpha}}{\frac{4 \sigma_{p}^{2}}{N}\left(\frac{N}{P_{p}}+\frac{L_{t_{p}}}{\alpha}\right)}\right)^{-L_{\Delta_{p}} L_{r_{p}}}
\end{aligned}
$$

where parameters $L_{\Delta_{p}}, \lambda_{i_{p}}, L_{r_{p}}$, and $\sigma_{p}^{2}$ for the primary-user in state $\nu=1$ are defined similarly to those of the relay-user in (13) and are independent from those of the secondary user except for the common factors $L_{t_{p}}, \alpha$, and $P_{p}$. We notice that while energy allocated to the data part of primary signal remains constant in the best-effort scheme, the PEP expression for a primary-user using pilot signals to reestimate channel conditions is dependent on $\alpha$, since both the primary and relay users must use the energy in these signals for channel estimation when $\nu=1$.

We now consider the power allocation problem for relays that instead optimize with respect to a rule limiting the SER experienced by the primary-user. From (16) and (18) the PEP expression under the two state model may be expressed as

$$
\begin{aligned}
& P(\mathbf{d} \rightarrow \mathbf{e})_{\mathbf{H}_{p}(t)} \leq \\
& P(\mathbf{d} \rightarrow \mathbf{e} \mid \nu=0)_{\mathbf{H}_{p}(t)} P(\nu=0)+ \\
& P(\mathbf{d} \rightarrow \mathbf{e} \mid \nu=1)_{\hat{\mathbf{H}}_{p}(t)} P(\nu=1) \\
& =\eta Q_{p}\left[R_{p}-(1-1 / \eta) S_{p}(\alpha)\right], \quad 0 \leq \alpha \leq 1
\end{aligned}
$$

where

$$
\begin{gathered}
Q_{p}=\left(\frac{1}{4 \sigma_{p}^{2}}\right)^{-L_{\Delta_{p}} L_{r_{p}}}\left(\prod_{i=1}^{L_{\Delta_{p}}} \lambda_{i_{p}}\right)^{-L_{r_{p}}} \\
R_{p}=P_{p}^{-L_{\Delta_{p}} L_{r_{p}}}(\alpha)=\left(\frac{1+\frac{\sigma_{s}^{2}}{\alpha}}{\frac{1}{N}\left(\frac{N}{P_{p}}+\frac{L_{t_{p}}}{\alpha}\right)}\right)^{-L_{\Delta_{p}} L_{r_{p}}}
\end{gathered}
$$

We evaluate this system under the constraint that the relay node must operate in a manor that maintains a minimum QoS with respect to the SER of its primary transmissions. The constraint for the primary user operating at a maximum allowable PEP is simply

$$
g_{p}(\alpha, \eta)=\eta Q_{p}\left[R_{p}-(1-1 / \eta) S_{p}(\alpha)\right], 0 \leq \alpha \leq 1
$$

Similarly, the constraint for the relay-user from (13) has a QoS threshold determined by maximum allowable PEP. This constraint is a function of the relay's choice of $\alpha$, or exactly

$$
\begin{aligned}
& g_{s}(\alpha)= \\
& \left(\prod_{i=1}^{L_{\Delta_{s}}} \lambda_{i_{s}}\right)^{-L_{r_{s}}}\left(\frac{1+\frac{\sigma_{s}^{2}}{\alpha}}{\frac{4 \sigma_{s}^{2}}{N}\left(\frac{N}{P_{p}-\alpha}+\frac{L_{t_{s}}}{\alpha}\right)}\right)^{-L_{\Delta_{s}} L_{r_{s}}}
\end{aligned}
$$

The optimal power allocation for the best-effort relay with respect to a maximum allowable SER for the primary-user is found by solving (23) for $\alpha_{p_{S E R}}$ under the constraint $g_{p}(\alpha, \eta) \geq G_{p}$

$$
\alpha_{p_{S E R}}^{*}=\frac{-P_{p}\left(L_{t_{p}}-\Psi\left(G_{p}, \eta\right) \sigma_{p}^{2}\right)}{N-P_{p} \Psi\left(G_{p}, \eta\right)}
$$

where

$$
\Psi\left(G_{p}, \eta\right)=\left(\frac{\eta P_{p}^{-L_{r_{p}} L_{\Delta_{p}}}-G_{p}\left(\frac{N}{4 \sigma_{p}^{2}}\right)^{L_{r_{p}} L_{\Delta_{p}}}}{\eta-1}\right)^{\frac{1}{L_{r_{p}} L_{\Delta_{p}}}}
$$

Power allocation using the SER-based allocation rule (25) with respect to $\eta$ is shown in Figure 2 for the threshold value $G_{p}=1.4 e^{-5}$. 
The terms $\alpha_{P 1}$ and $\alpha_{P 2}$ represent power allocations for the source and relay transmissions using a dedicated DF relay [1], and are provided for comparison. If power allocation $P_{2}$ of the relay transmission in the dedicated relay case is compared to the power allocated by a node that provides both primary transmission and relay services, these power allocations are sub-optimal under the best-effort delivery policy. We see that when channel conditions are stationary, i.e. $\eta \rightarrow 1$, the relay signal power $\alpha_{p}^{*}$ is equal to the dedicated-relay power $P_{2}$ when $\eta=.76$ for these parameters.

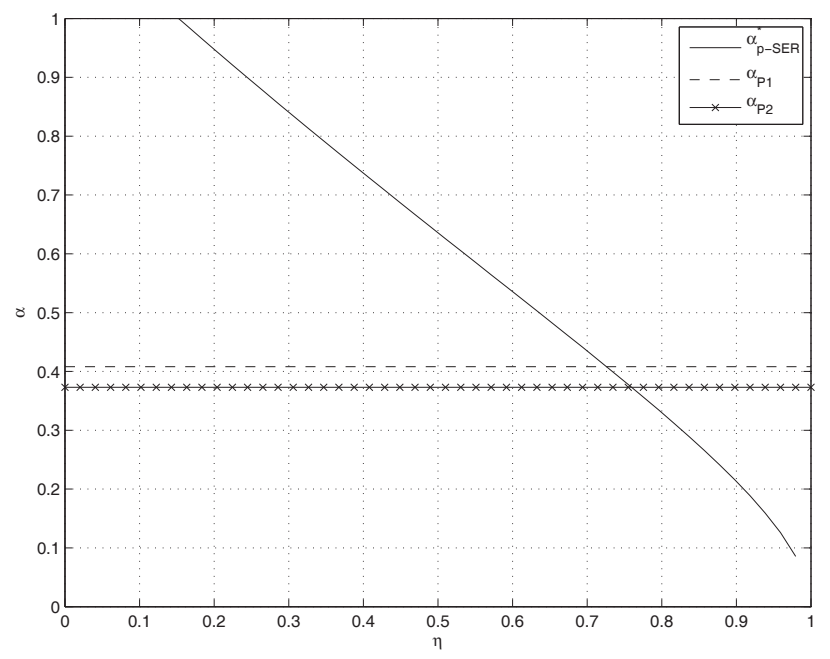

Fig. 2. $\alpha$ vs. $\eta$ - SER rule, for $G_{p}=1.4 e-5$

To observe the behavior of a relay node operating under the power constraint (25), Figure 3 demonstrates SER results of (23) and (24) with respect to the channel estimate confidence parameter $\eta$, for various values of $G_{p}$. We observe that cooperative diversity gains are obtainable for the relay-user under the SER rule as the relay diverts energy to relay-user transmissions. If too much energy is diverted from pilot signals, the SER for the relay-user suffers since its channel estimation performance is dependent on sufficient pilot energy, even though the signal strength of relayed signal has actually increased.

We expect that a power allocation rule optimizing with respect to a specific maximum primary-user SER threshold will exhibit a constant SER response over all channel stationary states when operating near optimum. This behavior is clearly discernible from Figure 3 , as SER for the primary-user is constant-valued for the entire range $0 \leq \eta \leq 1$. For comparison, the SER experienced by the primary and relay-users for a fixed power allocation policy of $\alpha=.5$ is presented in Figure 3.

The best-effort behavior of the system under this rule is apparent, as the primary-user consistently enjoys a general SER advantage over the relay-user. The effect of the SER threshold $G_{p}$ is shown in Figure 3. As $G_{p}$ increases, the relay will too readily divert energy from pilot signals and the SER of the primary and secondary users suffer accordingly. In general, decreasing $G_{p}$ has the effect of improving SER for both the primary and secondary users, at the cost of decreasing useful capacity for lower values of channel confidence (i.e. the SER curve for the relay-user is shifted to the right).

We conclude that the value for $G_{p}$ must also be carefully chosen with respect to the SNR experienced by both users under the SER-based QoS rule (25). In this example identical channel SNRs

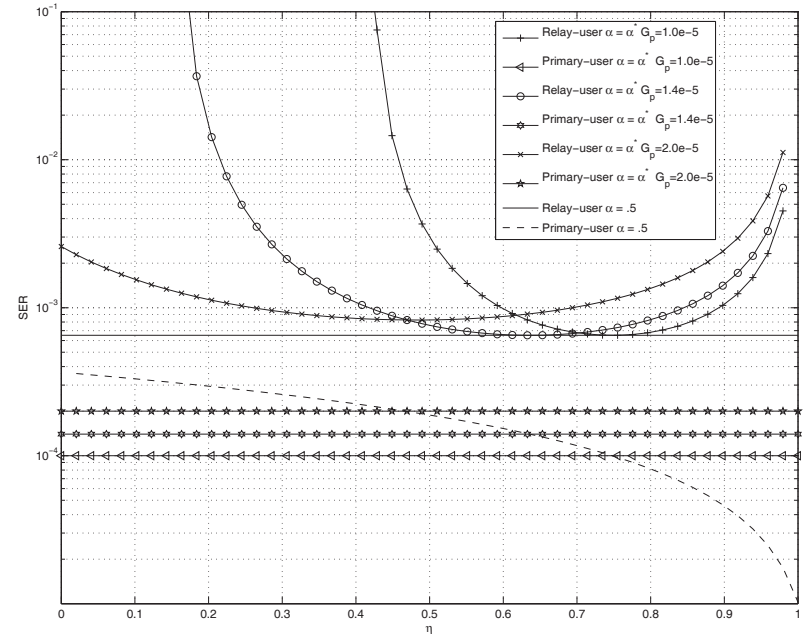

Fig. 3. SER vs. $\eta$ - SER rule, for values of $G_{p}$

were used for the primary and secondary users and a diversity gain is demonstrated for the relay-user when the relay employs cooperative relaying.

\section{CONCLUSION}

We have demonstrated that cooperative diversity may be achieved through a best-effort transmission policy. Using this method of delivery, energy may be re-purposed for relay transmissions at the discretion of the relay, while maintaining a minimum level of QoS for the node's primary mission. In on embodiment of best-effort delivery, it has been demonstrated that under stable channel conditions a node may re-distribute energy for relay services without significantly hindering primary-user transmissions, and while operating within its original transmission energy constraint.

\section{REFERENCES}

[1] K.J. Ray Liu, A.K. Sadek, W. Su, and A. Kwasinski, Cooperative Communications and Networking, Cambridge Press, 2009.

[2] C. Pirak, Z. Wang, K.J. Ray Liu, and S. Jitapunkul, "A databearing approach for pilot-embedding frameworks in space-time coded mimo systems," in IEEE Transactions on Signal Processing, October 2006, pp. 3966-3979.

[3] W. Su, Z. Safar, and K.J. Ray Liu, "Full-rate full-diversity spacefrequency codes with optimum coding advantage," in IEEE Transactions on Information Theory, January 2005, pp. 229-250.

[4] C. Pirak, Z. Wang, K.J. Ray Liu, and S. Jitapunkul, "Adaptive channel estimation using pilot-embedded data-bearing approach for mimo-ofdm systems," in IEEE Transactions on Signal Processing, December 2006, pp. 4706-4717.

[5] W. Su, A. Sadek, and K.J. Ray Liu, "Cooperative communication protocols in wireless networks: Performance analysis and optimum power allocation," Wireless Personal Communications, vol. 44, pp. 181-217, January 2008. 